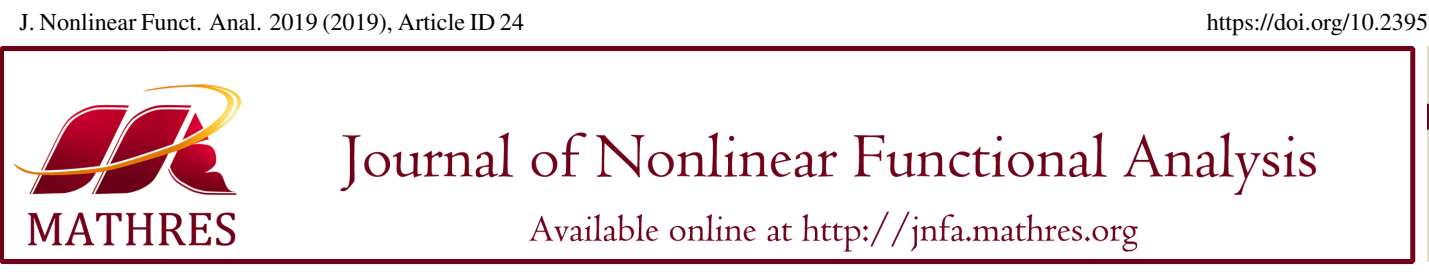

https://doi.org/10.23952/jnfa.2019.24

\title{
STRONGLY POSITIVE-LIKE OPERATORS AND EIGENVALUE CRITERIA FOR EXISTENCE AND NONEXISTENCE OF POSITIVE SOLUTIONS FOR $\alpha$-ORDER FRACTIONAL BOUNDARY VALUE PROBLEMS
}

\author{
ABDELHAMID BENMEZAIII ${ }^{1, *}$, SOUAD CHENTOUT ${ }^{1}$, JOHNNY HENDERSON ${ }^{2}$ \\ ${ }^{1}$ Faculty of Mathematics, USTHB, Algiers, Algeria \\ ${ }^{2}$ Department of Mathematics, Baylor University, Waco, Texas 76798-7328, USA
}

Abstract. In this paper, we investigate existence and nonexistence of positive solutions for the $\alpha$-order, where $2<\alpha \leq 3$, nonlinear fractional boundary value problem

$$
\left\{\begin{array}{l}
D^{\alpha} u(t)+f(t, u(t))=0,0<t<1 \\
u(0)=u^{\prime}(0)=u^{\prime}(1)=0
\end{array}\right.
$$

Keywords. Cone; Fixed point; Fractional differential equation; Monotone operator; Positive operator.

2010 Mathematics Subject Classification. 47H10, 26A33, 34B16.

\section{INTRODUCTION}

The study on the existence of positive solutions to fractional differential equations is important due to its numerous applications in various areas of physics, chemistry and engineering. Existence results has attracted much attention recently; see, for example, $[1,2,3,4,5,6,7]$ and the references therein.

In this paper, we investigate the existence and nonexistence of positive solutions for fractional boundary value problems having Carathéodory type nonlinearities. We are concerned in this paper with the following nonlinear fractional boundary value problem (FBVP for short)

$$
\left\{\begin{array}{l}
D^{\alpha} u(t)+f(t, u(t))=0,0<t<1, \\
u(0)=u^{\prime}(0)=u^{\prime}(1)=0,
\end{array}\right.
$$

where $2<\alpha \leq 3$ is a real number, $D^{\alpha}$ is the standard Riemann-Liouville derivative and $f:(0,1) \times$ $[0,+\infty) \rightarrow[0,+\infty)$ is an $\mathbb{L}^{1}$-Carathéodory function, that is,

- $f(\cdot, u)$ is a measurable function for all $u \geq 0$,

\footnotetext{
${ }^{*}$ Corresponding author.

E-mail addresses: aehbenmezai@gmail.com (A. Benmezaï) chentout@yahoo.fr (S. Chentout), Johnny_Henderson@baylor. edu (J. Henderson).
}

Received January 4, 2019; Accepted June 11, 2019.

(C)2019 Journal of Nonlinear Functional Analysis 
- $f(t, \cdot)$ is continuous for a.e. $t \in(0,1)$,

- for all $r>0$, there exists $\psi_{r} \in \mathbb{L}^{1}[0,1]$ such that $|f(t, u)| \leq \psi_{r}(t)$ for a.e. $t \in(0,1)$ and all $|u| \leq r$.

Existence and nonexistence results for positive solutions were established in $[8,9,10,11]$ under eigenvalue criteria for several classes of boundary value problems associated with ordinary differential equations. Optimality of these results (see [9, Remark 3.5]) motivate us to look for similar results. Evidently, the fractional nature of Problem (1.1) will give rise to some new difficulties.

Our main existence result (Theorem 3.8) will be obtained by means of a new fixed point theorem (Theorem 2.14). This new theorem states that if a positive mapping (mapping leaving invariant a cone in a Banach space) is approximately, upper bounded at $\infty$ and lower bounded at 0 (or the reverse) by positive compact linear operators, admits a fixed point whenever the approximative linear lower bound and upper bound are strongly positive-like operators having the spectral radius oppositely located with respect to 1 .

\section{A FIXED POINT THEOREM VIA STRONGLY POSITIVE-LIKE OPERATORS}

Let $(B,\|\cdot\|)$ be a real Banach space and let $K$ be an ordered cone in $B$ (that is, $K$ is a closed convex subset of $B$ with $K \cap(-K)=\left\{0_{B}\right\}$ and $(t K) \subset K$ for all $\left.t \geq 0\right)$. We denote by $L_{c}(B)$ the set of all linear compact self-mappings of $B$. It is well known that the cone $K$ induces a partial order in the Banach space $B$. We write for all $x, y \in B, x \preceq y$ if $y-x \in K, x \prec y$ if $y-x \in K$ and $y \neq x$ and $x \npreceq y$ if $y-x \notin K$. Notations $\succeq, \succ$, and $\nsucceq$ denote, respectively, the reverse situations.

Definition 2.1. An operator $L \in L_{c}(B)$ is said to be positive if $L(K) \subset K$ and strongly positive if int $(K) \neq$ $\emptyset$ and $L\left(K \backslash\left\{0_{B}\right\}\right) \subset \operatorname{int}(K)$.

Definition 2.2. Let $L \in L_{c}(B)$ be positive. $L$ is said to be lower bounded if inf $\{\|L u\|: u \in K \cap \partial B(0,1)\}>$ 0 , where $\partial B(0,1)$ is the boundary of the open unit ball.

Definition 2.3. Let $L \in L_{c}(B)$ be positive. A real number $\lambda$ is said to be a positive eigenvalue of $L$ if $\lambda>0$ and there exists $\phi \succ 0_{B}$ such that $L \phi=\lambda \phi$.

Definition 2.4. Let $T_{1}, T_{2}: K \rightarrow K$ be continuous mappings. We write $T_{1} \preceq T_{2}$ if $T_{1} x \preceq T_{2} x$ for all $x \in K$.

The proof of the main result of this section is based on the fixed point index theory. For sake of completeness, let us recall briefly some basic facts of the fixed point index theory. A subset $\Omega$ is called a retract of $B$ if there exists a continuous mapping $r: B \rightarrow \Omega$ such that $r(x)=x$ for all $x \in \Omega$. This kind of mapping is called a retraction.

Let $U$ be an open bounded subset of $\Omega$ such that $U \subset B\left(0_{B}, R\right)$. For any compact mapping $h: \bar{U} \rightarrow K$ with $h(x) \neq x$ for all $x \in \partial U$, the integer given by

$$
i(h, U, \Omega)=\operatorname{deg}\left(I-h \circ r, B\left(0_{B}, R\right) \cap r^{-1}(U), 0\right),
$$

where deg is the Leray-Schauder degree, is well defined and is called fixed point index.

The fixed point index has the following properties:

(1) Normality : $i(h, U, \Omega)=\mathbf{1}$ if $h(x)=x_{0} \in \bar{U}$ for all $x \in \bar{U}$

(2) Homotopy invariance : Let $H:[0,1] \times \bar{U} \rightarrow \Omega$ be a completely continuous mapping such that $H(t, x) \neq x$ for all $(t, x) \in[0,1] \times \partial U$. The integer $i(H(t, \cdot), U, \Omega)$ is independent of $t$. 
(3) Additivity :

$$
i(h, U, \Omega)=i\left(h, U_{1}, \Omega\right)+i\left(h, U_{2}, \Omega\right)
$$

whenever $U_{1}$ and $U_{2}$ are two disjoint open subsets of $U$ such that $h$ has no fixed point in $\bar{U} \backslash\left(U_{1} \cup U_{2}\right)$.

(4) Permanence : If $\Omega^{\prime}$ is a retract of $\Omega$ with $h(\bar{U}) \subset \Omega^{\prime}$ then

$$
i(h, U, \Omega)=i\left(h, U \cap \Omega^{\prime}, \Omega^{\prime}\right) .
$$

(5) Solution property : If $i(h, U, \Omega) \neq 0$, then $h$ admits a fixed point in $U$.

From Dugundji, we know that every nonempty closed convex set of $B$ is a retract of $B$. In particular, the cone $K$ is a retract of $B$. Let, $R>0, K_{R}=K \cap B\left(0_{B}, R\right)$, where $B\left(0_{B}, R\right)$ is the open ball of radius $R$ centered at $0_{B}$ and $\partial K_{R}$ is the boundary of $K_{R}$, and consider a compact mapping $h: \overline{K_{R}} \rightarrow K$ with $h x \neq x$ for all $x \in \partial K_{R}$. We will use in this work the following lemmas to provide fixed point index computations.

Lemma 2.5 ([12]). If $h x \nsucceq x$ for all $x \in \partial K_{R}$, then $i\left(h, K_{R}, K\right)=1$.

Lemma 2.6 ([12]). If $h x \not \leq x$ for all $x \in \partial K_{R}$, then $i\left(h, K_{R}, K\right)=0$.

For more details and proofs we refer the reader to [12].

The following theorem is known as the Krein-Rutman theorem. It presents a situation where the spectral radius $r(L)=\lim _{n \rightarrow \infty}\left\|L^{n}\right\|^{1 / n}$ of a positive linear compact operator $L$, is the unique positive eigenvalue of $L$.

Theorem 2.7 ([13]). Let $L \in L_{c}(B)$ be strongly positive. Then $r(L)$ is the unique positive eigenvalue of $L$.

Proposition 2.8 ([13]). Let $L \in L_{c}(B)$ be strongly positive and consider the nonhomogeneous equation

$$
\lambda u-L u=v
$$

with $v \succ 0_{B}$. Then Equation (2.1) has a unique positive solution if $\lambda>r(L)$ and no positive solution if $\lambda \leq r(L)$.

For all positive operators $L \in L_{c}(B)$, we introduce the subsets

$$
\begin{gathered}
\Lambda_{L}=\left\{\lambda \geq 0, \text { there exists } u \succ 0_{B} \text { such that } L u \succeq \lambda u\right\} \\
\Gamma_{L}=\left\{\lambda \geq 0, \text { there exists } u \succ 0_{B} \text { such that } L u \preceq \lambda u\right\} .
\end{gathered}
$$

Proposition 2.9. Let $L \in L_{c}(B)$ be strongly positive. Then

$$
r(L)=\sup \Lambda_{L}=\inf \Gamma_{L} .
$$

Proof. Note that $r(L) \in \Lambda_{L} \cap \Gamma_{L}$. We find from Theorem 2.7 in [14] that $\Lambda_{L}$ is bounded from above by $r(L)$, which leads to $\sup \Lambda_{L}=r(L)$.

Now, we show inf $\Gamma_{L} \geq r(L)$ by the contrary. Suppose that inf $\Gamma_{L}<r(L)$. Let $\theta \in\left(\inf \Gamma_{L}, r(L)\right)$ and $u \in K \backslash\left\{0_{B}\right\}$ be such that $L(u) \preceq \theta u$. In fact, we have that $L(u) \prec \theta u$. If $L(u)=\theta u$, then the uniqueness in Theorem 2.7 leads to the contradiction $r(L)=\theta<r(L)$. Thus, one has that the equation $\lambda u-L u=v$ has a positive solution for $\lambda=\theta<r(L)$ and $v=\theta u-L u$. This contradicts Proposition 2.8. This completes the proof. 
Definition 2.10. A positive operator $L \in L_{c}(B)$ is said to be a strongly positive-like operator if

$$
r(L)=\sup \Lambda_{L}=\inf \Gamma_{L}>0 .
$$

We can obtain from Lemmas 2.5 and 2.6 the following lemma.

Lemma 2.11. Let $L \in L_{c}(B)$ be a strongly positive-like operator. Then, for all $R>0$,

$$
i\left(L, K_{R}, K\right)=\left\{\begin{array}{l}
1, \text { if } r(L)<1, \\
0, \text { if } r(L)>1 .
\end{array}\right.
$$

Remark 2.12. We understand from the above lemma that if $L \in L_{c}(B)$ is a strongly positive-like operator, then $r(L)$ is a positive eigenvalue of $L$. Indeed, we have, for all $\gamma, R>0$,

$$
i\left(\gamma L, K_{R}, K\right)=\left\{\begin{array}{l}
1, \text { if } r(\gamma L)=\gamma r(L)<1 \\
0, \text { if } r(\gamma L)=\gamma r(L)>1 .
\end{array}\right.
$$

Proposition 2.13. Let $T: K \rightarrow K$ be a continuous mapping and let $L \in L_{c}(B)$ be a strongly positive-like operator. If either

$$
r(L)>1 \text { and } T u \succeq \text { Lu for all } u \in K
$$

or

$$
r(L)<1 \text { and } T u \preceq \text { Lu for all } u \in K,
$$

then $T$ has no positive fixed point.

Proof. We present the proof in the case of (2.2) and the other one can be obtained similarly. On the contrary, we suppose there exists $u \succ 0_{B}$ such that $T u=u$. In this case, we have that $u=T u \succeq L u, 1 \in \Gamma_{L}$ and $r(L)=\inf \Gamma_{L} \leq 1$. This contradicts the hypothesis $r(L)>1$ in (2.2).

Theorem 2.14. Let $T: K \rightarrow K$ be a completely continuous mapping and assume that there exist two strongly positive-like operators $L_{1}, L_{2} \in L_{c}(B)$ and two functions $F_{1}, F_{2}: K \rightarrow K$ such that $L_{1}$ is lower bounded on $K, r\left(L_{2}\right)<1<r\left(L_{1}\right)$ and for all $u \in K$

$$
L_{1} u-F_{1} u \preceq T u \preceq L_{2} u+F_{2} u .
$$

If either

$$
F_{1} u=\circ(\|u\|) \text { as } u \rightarrow \infty \text { and } F_{2} u=\circ(\|u\|) \text { as } u \rightarrow 0
$$

or

$$
F_{1} u=\circ(\|u\|) \text { as } u \rightarrow 0 \text { and } F_{2} u=\circ(\|u\|) \text { as } u \rightarrow \infty,
$$

then $T$ has a positive fixed point.

Proof. We present the proof in the case where (2.4) holds and the other case can be obtained similarly. We have to prove existence of $0<r<R$ such that $i\left(T, K_{r}, K\right)=1$ and $i\left(T, K_{R}, K\right)=0$. In such a situation, additivity and solution properties of the fixed point index imply that

$$
i\left(T, K_{R} \backslash \overline{K_{r}}, K\right)=i\left(T, K_{R}, K\right)-i\left(T, K_{r}, K\right)=-1
$$

and $T$ has a positive fixed point $u$ with $r<\|u\|<R$.

Consider the function $H_{0}:[0,1] \times K \rightarrow K$ defined by

$$
H_{0}(t, u)=(1-t) T u+t L_{2} u
$$


and let us prove existence of $r>0$ small enough such that, for all $t \in[0,1]$, equation $H_{0}(t, u) \neq u$ in $\partial K_{r}$. On the contrary, we suppose that, for all integers $n \geq 1$, there exist $t_{n} \in[0,1]$ and $u_{n} \in \partial K_{1 / n}$ such that

$$
u_{n}=\left(1-t_{n}\right) T u_{n}+t_{n} L_{2} u_{n} .
$$

Note that $v_{n}=u_{n} /\left\|u_{n}\right\| \in \partial K_{1}$ and satisfies

$$
v_{n}=\left(1-t_{n}\right)\left(T u_{n} /\left\|u_{n}\right\|\right)+t_{n} L_{2} v_{n}
$$

and

$$
L_{2} v_{n}=\left(1-t_{n}\right) L_{2}\left(T u_{n} /\left\|u_{n}\right\|\right)+t_{n} L_{2}\left(L_{2} v_{n}\right) .
$$

Because of the compactness of $L_{2}$, there exists a subsequence $\left(v_{n_{k}}\right)$ such that $\left(L_{2} v_{n_{k}}\right)$ converges to $v \in$ $K \backslash\left\{0_{B}\right\}$. Indeed, if $v=0_{B}$, we obtain, from the inequality

$$
\left(T u_{n} /\left\|u_{n}\right\|\right) \preceq L_{2}\left(v_{n}\right)+\left(F_{2} u_{n} /\left\|u_{n}\right\|\right)
$$

and the hypothesis $F_{2} u=\circ(\|u\|)$ as $u \rightarrow 0$ in (2.4), that $\lim \left(T u_{n_{k}} /\left\|u_{n_{k}}\right\|\right)=0_{B}$. This implies from (2.6) that

$$
0_{B}=\lim v_{n_{k}}=\left(1-t_{n_{k}}\right)\left(T u_{n_{k}} /\left\|u_{n_{k}}\right\|\right) t_{n_{k}} L_{2} v_{n_{k}} .
$$

This contradicts $\left\|v_{n_{k}}\right\|=1$. Therefore, passing to the limit in (2.7), we obtain $v \preceq L_{2}(v)$ and $1 \leq \sup \Lambda_{L_{2}}=$ $r\left(L_{2}\right)$, which contradicts $r\left(L_{2}\right)<1$. Thus, there exists $r>0$ small enough such that $H_{0}(t, u) \neq u$ for all $t \in[0,1]$ and $u \in \partial K_{r}$ and for such a radius $r>0$, homotopy property of the fixed point index and Lemma 2.11 lead to

$$
i\left(T, K_{r}, K\right)=i\left(H_{0}(0, \cdot), K_{r}, K\right)=i\left(H_{0}(1, \cdot), K_{r}, K\right)=i\left(L_{2}, K_{r}, K\right)=1 .
$$

Similarly, consider the function $H_{\infty}:[0,1] \times K \rightarrow K$ defined by

$$
H_{\infty}(t, u)=(1-t) T u+t L_{1} u .
$$

Let us prove the existence of $R>0$ large enough such that, for all $t \in[0,1]$, equation $H_{\infty}(t, u) \neq u$ in $\partial K_{R}$. On the contrary, we suppose that, for all integers $n \geq 1$, there exist $t_{n} \in[0,1]$ and $u_{n} \in \partial K_{n}$ such that

$$
u_{n}=\left(1-t_{n}\right) T u_{n}+t_{n} L_{1} u_{n}
$$

Note that $v_{n}=u_{n} /\left\|u_{n}\right\| \in \partial K_{1}$ satisfies

$$
v_{n}=\left(1-t_{n}\right)\left(T u_{n} /\left\|u_{n}\right\|\right)+t_{n} L_{1} v_{n}
$$

and then

$$
L_{1} v_{n}=\left(1-t_{n}\right) L_{1}\left(T u_{n} /\left\|u_{n}\right\|\right)+t_{n} L_{1}\left(L_{1} v_{n}\right) .
$$

Because of the compactness and the lower boundedness of $L_{1}$, there exists a subsequence $\left(v_{n_{k}}\right)$ such that $\left(L_{1} v_{n_{k}}\right)$ converges to some $w$ and $\|w\|=\lim \left\|L_{1} v_{n}\right\| \geq c_{L_{1}, K}>0$, where

$$
c_{L_{1}, K}=\inf \left\{\left\|L_{1} u\right\|, u \in \partial K_{1}\right\} .
$$

Thus, we obtain from (2.8) the estimates

$$
\begin{aligned}
L_{1} v_{n_{k}} & =\left(1-t_{n_{k}}\right) L_{1}\left(T u_{n_{k}} /\left\|u_{n}\right\|\right)+t_{n_{k}} L_{1}\left(L_{1} v_{n_{k}}\right) \\
& \succeq L_{1}\left(L_{1} v_{n_{k}}\right)-\left(1-t_{n_{k}}\right) L_{1}\left(F_{1} u_{n_{k}} /\left\|u_{n}\right\|\right) .
\end{aligned}
$$

Then from the hypothesis $F_{1} u=\circ(\|u\|)$ as $u \rightarrow \infty$ in (2.4), $w \succeq L_{1} w$ and $1 \in \Gamma_{L_{1}}$. This leads to the contradiction $r\left(L_{1}\right)>1 \geq \inf \Gamma_{L_{1}}=r\left(L_{1}\right)$. 
Thus, there exists $R>0$ large such that $H_{\infty}(t, u) \neq u$ for all $t \in[0,1]$ and $u \in \partial K_{R}$ and for such a radius $R>0$, homotopy property of the fixed point index and Lemma 2.11 imply that

$$
i\left(T, K_{R}, K\right)=i\left(H_{\infty}(0, \cdot), K_{R}, K\right)=i\left(H_{\infty}(1, \cdot), K_{R}, K\right)=i\left(L_{1}, K_{R}, K\right)=0 .
$$

This completes the proof.

\section{EIGENVALUE CRITERIA FOR THE EXISTENCE AND NONEXISTENCE OF POSITIVE SOLUTIONS} FOR $\alpha$-ORDER $(2<\alpha \leq 3)$ FRACTIONAL BVPS WITH CARATHÉODORY NONLINEARITIES

3.1. Preliminaries. Now, let us recall some basic facts related to the theory of fractional differential equations. Let $\beta$ be a nonnegative real number. The Riemann-Liouville fractional integral of order $\beta$ of a function $f:(0,+\infty) \rightarrow \mathbb{R}$ is defined by

$$
I_{0^{+}}^{\beta} f(t)=\left\{\begin{array}{l}
\frac{1}{\Gamma(\beta)} \int_{0}^{t}(t-s)^{\beta-1} f(s) d s, \text { if } \beta>0, \\
f(t), \text { if } \beta=0,
\end{array}\right.
$$

where $\Gamma(\beta)$ is the Gamma function provided that the right side is pointwise defined on $(0,+\infty)$. For example, we have, for any real $\sigma>-1$,

$$
I_{0^{+}}^{\beta} t^{\sigma}=\frac{\Gamma(\sigma+1)}{\Gamma(\sigma+\beta+1)} t^{\sigma+\beta} .
$$

The Riemann-Liouville fractional derivative of order $\beta$ of a continuous function $f:(0,+\infty) \rightarrow \mathbb{R}$ is given by

$$
D_{0^{+}}^{\beta} f(t)=\frac{1}{\Gamma(n-\beta)}\left(\frac{d}{d t}\right)^{n} \int_{0}^{t} \frac{f(s)}{(t-s)^{\beta-n+1}} d s,
$$

where $n=[\beta]+1,[\beta]$ denotes the integer part of the number $\beta$ provided that the right side is pointwise defined on $(0, \infty)$. As a basic example, for $\sigma>-1$,

$$
D_{0^{+}}^{\beta} t^{\sigma}=\frac{\Gamma(\sigma+1)}{\Gamma(\sigma-\beta+1)} t^{\sigma-\beta} .
$$

Thus, if $u \in C(0,1) \cap L^{1}(0,1)$, then fractional differential equation $D_{0^{+}}^{\beta} u(t)=0$ has

$$
u(t)=\sum_{i=1}^{i=[\beta]+1} c_{i} t^{\beta-i}
$$

$c_{i} \in \mathbb{R}$, as the unique solution and if $u$ has a fractional derivative of order $\beta$ in $C(0,1) \cap \mathbb{L}^{1}(0,1)$, then

$$
I_{0^{+}}^{\beta} D_{0^{+}}^{\beta} u(t)=u(t)+\sum_{i=1}^{i=[\beta]+1} c_{i} t^{\beta-i}, c_{i} \in \mathbb{R},
$$

and

$$
D_{0^{+}}^{\alpha} I_{0^{+}}^{\beta} u(t)=I_{0^{+}}^{\beta-\alpha} u(t) \text { if } \beta \geq \alpha>0 .
$$

For a detailed presentation on fractional differential equations, we refer the read to [15] or [16].

Now, we introduce some spaces and operators which are needed for the proof of the main results in this paper. Throughout this section, we let

$$
\mathbb{L}^{1}=\left\{m:(0,1) \rightarrow \mathbb{R}: m \text { is measurable and } \int_{0}^{1}|m(t)| d t<\infty\right\}
$$

be equipped with its norm $\|\cdot\|_{1}$ and let $E$ be the Banach space of all continuous functions defined on $[0,1]$, endowed with its sup-norm denoted $\|\cdot\|_{\infty}$. 
We also define the spaces $X$ and $Y$ by

$$
\begin{aligned}
X & =\left\{u \in C[0,1]: \lim _{t \rightarrow 0} \frac{u(t)}{t^{\alpha-1}} \text { exists }\right\} \text { and } \\
Y & =\left\{u \in X: \frac{u(t)}{t^{\alpha-1}} \in C^{1}[0,1]\right\} .
\end{aligned}
$$

Throughout this section, the linear spaces $X$ and $Y$ are equipped respectively with the norms

$$
\begin{aligned}
& \|u\|_{X}=\sup \left\{\left|\frac{u(t)}{t^{\alpha-1}}\right|: t \in[0,1]\right\} \text { and } \\
& \|u\|_{Y}=\|u\|_{X}+\sup \left\{\left|\left(\frac{u(t)}{t^{\alpha-1}}\right)^{\prime}\right|: t \in[0,1]\right\}
\end{aligned}
$$

It is easy to check that $\left(X,\|\cdot\|_{X}\right)$ and $\left(Y,\|\cdot\|_{Y}\right)$ are Banach spaces, and $i$ the embedding of $C^{1}[0,1]$ into $E$ and $j$ the embedding of $Y$ into $X$ are compact.

$E^{+}$is the cone of nonnegative functions in $E$ and, for fixed $0<\delta<1, P$ is the cone in $E$ defined by

$$
P=\left\{u \in E^{+}: u(t) \geq \delta^{\alpha-1}\|u\|_{\infty} \text { for all } t \in[\delta, 1]\right\} .
$$

$X^{+}$is the natural cone in $X$, i.e., $X^{+}=\{u \in X: u(t) \geq 0$ for all $t \in[0,1]\}$.

$$
\begin{aligned}
& \mathbb{L}_{+}^{1}=\left\{m \in \mathbb{L}^{1}(0,1): m(t) \geq 0 \text { a.e. } t \in(0,1)\right\}, \\
& \mathbb{L}_{++}^{1}=\left\{m \in \mathbb{L}_{+}^{1}: m>0 \text { in a subset of positive measure }\right\} .
\end{aligned}
$$

We need to introduce the following subset of $X$.

$$
S=\left\{u \in X: u(t)>0 \text { for all } t \in(0,1] \text { and } \lim _{t \rightarrow 0} \frac{u(t)}{t^{\alpha-1}}>0\right\} .
$$

Lemma 3.1. $S$ is open in $X$.

Proof. We have to show that $X \backslash S$ is a closed set in $X$. Note that $X \backslash S=F_{1} \cup F_{2}$, where

$$
\begin{aligned}
& F_{1}=\{u \in X: u(t) \leq 0 \text { for some } t \in(0,1]\} \\
& F_{2}=\left\{u \in X: \lim _{t \rightarrow 0} \frac{u(t)}{t^{\alpha-1}} \leq 0\right\} .
\end{aligned}
$$

Clearly, $F_{2}$ is a closed set in $X$. Thus, let $\left(u_{n}\right) \subset F_{1}$ converging to $u$ in $X,\left(t_{n}\right) \subset(0,1)$ with $\lim t_{n}=\bar{t} \in[0,1]$ and $u_{n}^{\prime}\left(t_{n}\right) \leq 0$. We distinguish the following two cases.

i) $\bar{t} \in(0,1]$. In this case, we have $u(\bar{t})=\lim _{n \rightarrow \infty} u_{n}\left(t_{n}\right) \leq 0$. This shows $u \in F_{1}$.

ii) $\bar{t}=0$. In this case, we have $u \in F_{2}$. Indeed, if

$$
\lim _{t \rightarrow 0} \frac{u(t)}{t^{\alpha-1}}=l>0,
$$

then there exists an integer $n_{0}$ such that, for all $t \in(0,1]$,

$$
\frac{u(t)}{t^{\alpha-1}}-\frac{l}{4} \leq \frac{u_{n}(t)}{t^{\alpha-1}} \leq \frac{u(t)}{t^{\alpha-1}}+\frac{l}{4} .
$$

Moreover, there exists $\delta>0$ such that, for all $t \in(0, \delta]$,

$$
-\frac{l}{4} \leq \frac{u(t)}{t^{\alpha-1}}-l \leq \frac{l}{4}
$$


Thus, let $n_{1}$ be such that $t_{n} \in(0, \delta]$ for all $n \geq n_{1}$. We have, for all integer $n \geq \max \left(n_{0}, n_{1}\right)$, the contradiction

$$
\begin{aligned}
0 & \geq u_{n}\left(t_{n}\right)=\left(t_{n}^{\alpha-1}\right)\left(\frac{u_{n}(t)}{t^{\alpha-1}}\right) \geq\left(t_{n}^{\alpha-1}\right)\left(\frac{u(t)}{t^{\alpha-1}}-\frac{l}{4}\right) \\
& \geq \frac{l}{2}\left(t_{n}^{\alpha-1}\right)>0 .
\end{aligned}
$$

This ends the proof.

Let $G:[0,1] \times[0,1] \rightarrow \mathbb{R}$ be a continuous function defined by

$$
G(t, s)=\frac{1}{\Gamma(\alpha)}\left\{\begin{array}{l}
t^{\alpha-1}(1-s)^{\alpha-2}-(t-s)^{\alpha-1}, \text { if } 0 \leq s \leq t \leq 1 \\
t^{\alpha-1}(1-s)^{\alpha-2}, \text { if } 0 \leq t \leq s \leq 1
\end{array}\right.
$$

The function $G$ has the following properties:

$$
\begin{gathered}
G(t, s)>0 \text { for all } t, s \in(0,1), \\
G(t, s) \leq G(1, s) \text { for all } t, s \in[0,1], \\
G(t, s) \geq \delta^{\alpha-1} G(1, s) \text { for all } t \in[\delta, 1] \text { and all } s \in[0,1] .
\end{gathered}
$$

Lemma 3.2. Consider, for $h \in \mathbb{L}^{1}(0,1)$, the following FBVP

$$
\left\{\begin{array}{l}
D_{0^{+}}^{\alpha} u(t)+h(t)=0, t \in(0,1) \\
u(0)=u^{\prime}(0)=u^{\prime}(1)=0
\end{array}\right.
$$

Then $u$ is a solution to FBVP (3.8) if and only if $u=£$, where $£: \mathbb{L}^{1}(0,1) \rightarrow C^{1}[0,1]$ with $£ h(t)=$ $\int_{0}^{1} G(t, s) h(s) d s$.

Proof. Let $u$ be a solution to FBVP (3.8). We find by (3.3) that

$$
u(t)=-\frac{1}{\Gamma(\alpha)} \int_{0}^{t}(t-s)^{\alpha-1} h(s) d s+c_{1} t^{\alpha-1}+c_{2} t^{\alpha-2}+c_{3} t^{\alpha-3},
$$

for some $c_{1}, c_{2}, c_{3} \in \mathbb{R}$. Then, the the boundary conditions $u(0)=u^{\prime}(0)=u^{\prime}(1)=0$ leads to $c_{2}=c_{3}=0$ and

$$
c_{1}=\frac{1}{\Gamma(\alpha)} \int_{0}^{1}(1-s)^{\alpha-2} h(s) d s
$$

Thus,

$$
\begin{aligned}
u(t) & =-\frac{1}{\Gamma(\alpha)} \int_{0}^{t}(t-s)^{\alpha-1} h(s) d s+\frac{1}{\Gamma(\alpha)} \int_{0}^{1} t^{\alpha-1}(1-s)^{\alpha-2} h(s) d s \\
& =\frac{1}{\Gamma(\alpha)}\left(\int_{0}^{t}\left(t^{\alpha-1}(1-s)^{\alpha-2}-(t-s)^{\alpha-1}\right) h(s) d s+\int_{t}^{1} t^{\alpha-1}(1-s)^{\alpha-2} h(s) d s\right. \\
& =\int_{0}^{1} G(t, s) h(s) d s=£ h(t) .
\end{aligned}
$$

Conversely, if

then

$$
u(t)=£ h(t)=\int_{0}^{1} G(t, s) h(s) d s
$$

$$
u(t)=-I_{0^{+}}^{\alpha} h(t)+\frac{t^{\alpha-1}}{\Gamma(\alpha)} \int_{0}^{1}(1-s)^{\alpha-2} h(s) d s .
$$


From (3.4), one has $D_{0^{+}}^{\alpha} u(t)=-h(t)$. Since $G(0, s)=0$ for all $s \in[0,1]$, we have

$$
u(0)=\int_{0}^{1} G(0, s) h(s) d s=0 .
$$

Moreover, from

$$
u^{\prime}(t)=\frac{(\alpha-1) t^{\alpha-2}}{\Gamma(\alpha)} \int_{0}^{1}(1-s)^{\alpha-2} h(s) d s-\frac{(\alpha-1)}{\Gamma(\alpha)} \int_{0}^{t}(t-s)^{\alpha-2} h(s) d s
$$

we obtain $u^{\prime}(0)=u^{\prime}(1)=0$. The proof is complete.

Let, for $m \in \mathbb{L}_{++}^{1}, L_{m}: E \rightarrow E$ and $L_{m}^{X}: X \rightarrow X$ be the linear continuous operator such that $L_{m}^{X} u=L_{m} u$ for all $u \in X$ and for $u \in E L_{m} u(t)=\int_{0}^{1} G(t, s) m(s) u(s) d s$.

Lemma 3.3. For all $m \in \mathbb{L}_{++}^{1}$, the operator $L_{m}$ is compact and positive. Moreover, $L_{m}\left(E^{+}\right) \subset P$.

Proof. Note that $L_{m}=£_{m} \circ i$, where $£_{m}: E \rightarrow C^{1}[0,1]$ is defined for $u \in E$ by

$$
£_{m} u(t)=\int_{0}^{1} G(t, s) m(s) u(s) d s .
$$

Since the linear operator $£_{m}$ is continuous and $i$ is compact, the operator $L_{m}$ is compact. Let $u \in E^{+}$, we have from (3.5) that, for all $t \in[0,1]$,

$$
L_{m} u(t)=\int_{0}^{1} G(t, s) m(s) u(s) d s \geq 0 .
$$

Thus $L_{m} u \in E^{+}$and the operator $L_{m}$ is positive. We prove now that $L_{m}\left(E^{+}\right) \subset P$. Letting $v \in E^{+}$, we have from (3.7) and (3.6) of function $G$ that, for all $t \in[\delta, 1]$,

$$
L_{m} v(t)=\int_{0}^{1} G(t, s) m(s) v(s) d s \geq \delta^{\alpha-1} \int_{0}^{1} G(1, s) m(s) v(s) d s=\delta^{\alpha-1}\left\|L_{m} v\right\| .
$$

This proves that $L_{m} v \in P$ and $L_{m}\left(E^{+}\right) \subset P$. This completes the proof.

Lemma 3.4. For all $m \in \mathbb{L}_{++}^{1}$, $L_{m}$ is a strongly positive-like operator which is lower bounded on the cone $P$.

Proof. First, we prove that, for all $m \in \mathbb{L}_{+}^{1} \cap C[0,1], L_{m}^{X}$ is a strongly positive operator. We begin by proving the compactness of $L_{m}^{X}$. Let $\left(m_{n}\right)$ be a sequence in $C_{c}[0,1]$ converging to $m$ in $\mathbb{L}^{1}$ and note that for all integer $n, L_{m_{n}}^{X}=j \circ £_{m_{n}}^{Y}$, where $£_{m_{n}}^{Y}: X \rightarrow Y$ is defined for $u \in X$ by

$$
\begin{aligned}
£_{m_{n}}^{Y} u(t) & =\frac{1}{\Gamma(\alpha)}\left(\int_{0}^{1} t^{\alpha-1}(1-s)^{\alpha-1} m_{n}(s) u(s) d s-\int_{0}^{t}(t-s)^{\alpha-1} m_{n}(s) u(s) d s\right) \\
& =\frac{t^{\alpha-1}}{\Gamma(\alpha)}\left(\int_{0}^{1}(1-s)^{\alpha-1} m_{n}(s) u(s) d s-t \int_{0}^{1}(1-s)^{\alpha-1} m_{n}(t s) u(t s) d s\right) .
\end{aligned}
$$

The compactness of $L_{m_{n}}^{X}$ follows from that of $j$ once we prove that the linear operator $\AA_{m_{n}}^{Y}$ is continuous. Letting $u \in X, v=£_{m_{n}}^{Y} u$ and $w(t)=\frac{v(t)}{t^{\alpha-1}}$, one has

$$
w^{\prime}(t)=-\frac{(\alpha-1)}{\Gamma(\alpha)}\left(\int_{0}^{1} s(1-s)^{\alpha-2} m_{n}(t s) u(t s) d s\right) .
$$

It is easy to check that

$$
\left\|{£_{m_{n}}^{Y}} u\right\|_{Y}=\|w\|_{\infty}+\left\|w^{\prime}\right\|_{\infty} \leq\left\|m_{n}\right\|_{\infty}\|u\|_{\infty} \leq C(\alpha)\left\|m_{n}\right\|_{\infty}\|u\|_{X},
$$


where

$$
C(\alpha)=\frac{1}{\Gamma(\alpha)}\left(2 \int_{0}^{1}(1-s)^{\alpha-1} d s+(\alpha-1) \int_{0}^{1}(1-s)^{\alpha-2} d s\right) .
$$

This shows that $f_{m_{n}}^{Y}$ is continuous and $L_{m_{n}}^{X}$ is compact. Because $L_{m_{n}}^{X} \rightarrow L_{m}^{X}$ in operator norm, we have that $L_{m}^{X}$ is compact.

Now, let $u \in X^{+} \backslash\left\{0_{X}\right\}$ and $v=L_{m}^{X} u$. We have from (3.5) and (3.6) that, for all $t \in(0,1]$,

$$
v(t)=\int_{0}^{1} G(t, s) m(s) u(s) d s>0 .
$$

We obtain after simple calculations that

$$
\begin{aligned}
\left|\frac{v(t)}{t^{\alpha-1}}-\frac{1}{\Gamma(\alpha)} \int_{0}^{1}(1-s)^{\alpha-2} m(s) u(s) d s\right| & \leq \frac{1}{\Gamma(\alpha) t^{\alpha-1}} \int_{0}^{t}(t-s)^{\alpha-1} m(s) u(s) d s \\
& \leq \frac{\|u\|_{X}}{\Gamma(\alpha)} \int_{0}^{t}(1-s)^{\alpha-1} m(s) d s,
\end{aligned}
$$

which leads to

$$
\lim _{t \rightarrow 0} \frac{v(t)}{t^{\alpha-1}}=\frac{1}{\Gamma(\alpha)} \int_{0}^{1}(1-s)^{\alpha-2} m(s) u(s) d s>0 .
$$

The above estimates show that $L_{m}^{X} u \in S \subset \operatorname{int}\left(X^{+}\right)$and

$$
L_{m}^{X}\left(X^{+} \backslash\left\{0_{X}\right\}\right) \subset S \subset \operatorname{int}\left(X^{+}\right) .
$$

Then, Proposition 2.9 leads to

$$
r\left(L_{m}^{X}\right)=\sup \left(\Lambda_{L_{m}^{X}}\right)=\inf \left(\Gamma_{L_{m}^{X}}\right)
$$

where

$$
\begin{aligned}
& \Lambda_{L_{m}^{X}}=\left\{\lambda \geq 0: \text { there exists } u \in X^{+} \backslash\left\{0_{X}\right\} \text { such that } L_{m}^{X} u \succeq \lambda u\right\} \\
& \Gamma_{L_{m}^{X}}=\left\{\lambda \geq 0: \text { there exists } u \in X^{+} \backslash\left\{0_{X}\right\} \text { such that } L_{m}^{X} u \preceq \lambda u\right\} .
\end{aligned}
$$

Clearly, we have $\Lambda_{L_{m}^{X}} \subset \Lambda_{L_{m}}$ and $\Gamma_{L_{m}^{X}} \subset \Gamma_{L_{m}}$. So, let us prove that $\Lambda_{L_{m}^{X}}=\Lambda_{L_{m}}$ and $\Gamma_{L_{m}^{X}}=\Gamma_{L_{m}}$. To this aim, let $\lambda \geq 0$ and $u \in E^{+} \backslash\left\{0_{E}\right\}$ be such that $L_{m} u \succeq \lambda u$. Then, $U=L_{m} u \in X^{+} \backslash\left\{0_{X}\right\}$,

$$
L_{m}^{X} U=L_{m} L_{m} u \succeq \lambda L_{m} u=\lambda U
$$

and $\lambda \in \Lambda_{L_{m}^{X}}$. So, we have proved that $\Lambda_{L_{m}^{X}}=\Lambda_{L_{m}}$ In a similar way, we can also obtain that $\Gamma_{L_{m}^{X}}=\Gamma_{L_{m}}$. Thus,

$$
r\left(L_{m}^{X}\right)=\sup \left(\Lambda_{L_{m}^{X}}\right)=\sup \left(\Lambda_{L_{m}}\right)=\inf \left(\Gamma_{L_{m}^{X}}\right)=\inf \left(\Gamma_{L_{m}}\right)=r\left(L_{m}\right) .
$$

Now, we have, for all $u \in P$,

$$
\left\|L_{m} u\right\|=L_{m} u(1)=\int_{0}^{1} G(1, s) m(s) u(s) d s \geq\left(\delta^{\alpha-1} \int_{0}^{1} G(1, s) m(s) \delta^{\alpha-1} d s\right)\|u\|,
$$

which proves that $L_{m}$ is lower bounded on the cone $P$. This ends the proof.

Consider the operators $F: E^{+} \rightarrow \mathbb{L}_{+}^{1}$ and $T: E^{+} \rightarrow E$ defined by

$$
F u(t)=f(t, u(t)), \text { and } T=i \circ £ \circ F .
$$

\section{Lemma 3.5. We have that}

i) $T$ is completely continuous,

ii) $T\left(E^{+}\right) \subset P$ and

iii) $u \in E$ is a positive solution to Problem (1.1) if and only if $u$ is a fixed point of $T$. 
Proof. i) Note that the fact that the nonlinearity $f$ is an $\mathbb{L}^{1}$-Caratheodory function makes the mapping $F$ continuous and bounded (maps bounded sets of $E^{+}$into bounded sets in $\mathbb{L}^{1}(0,1)$ ). This, together with the continuity of the linear operator $£$ and the compactness of the embedding $i$, makes of $T$ a completely continuous mapping.

ii) This is due to properties (3.5)-(3.7) of function $G$.

iii) It follows from Lemma 3.2.

The proof is complete.

3.2. Mains results. Letting $m \in \mathbb{L}_{++}^{1}$. For $2<\alpha \leq 3$, we consider the linear FBVP

$$
\left\{\begin{array}{l}
D^{\alpha} u(t)+\mu m(t) u(t)=0, \text { a.e. } t \in(0,1), \\
u(0)=u^{\prime}(0)=u^{\prime}(1)
\end{array}\right.
$$

where $\mu$ is a real parameter.

Proposition 3.6. For all $m \in \mathbb{L}_{++}^{1}, F B V P(3.9)$ admits a unique positive eigenvalue $\mu_{\alpha}(m)$.

Proof. It follows from iii) of Lemma 3.5 that the pair $(\mu, u)$ is a solution of FBVP (3.9) if and only if $L_{m} u=\mu^{-1} u$. Hence, we conclude by Lemma 3.4 that $\mu^{-1}=r\left(L_{m}\right)$ is the unique positive eigenvalue of $L_{m}$. Then $\mu_{\alpha}(m)=1 / r\left(L_{m}\right)$ is the unique positive eigenvalue of FBVP (3.9).

Proposition 3.7. Assume that there exists $m \in \mathbb{L}_{+}^{1}$ such that one of the following hypotheses

$$
\begin{aligned}
& \mu_{\alpha}(m)<1 \text { and } f(t, u) \geq m(t) u \text { for all } u \geq 0 \text { and } a . \text { e. } t \in(0,1), \\
& \mu_{\alpha}(m)>1 \text { and } f(t, u) \leq m(t) u \text { for all } u \geq 0 \text { and a. } e . t \in(0,1),
\end{aligned}
$$

is satisfied. Then Problem (1.1) has no positive solutions.

Proof. If (3.10) holds, then $f(t, u) \geq m(t) u$. In this case, $T u \geq L_{m} u$ and $L_{m}$ is a strongly positive-like operator with $r\left(L_{m}\right)=1 / \mu_{\alpha}(m)>1$. Thus, Hypothesis (2.2) is satisfied and Proposition 2.13 guarantees that $T$ has no positive fixed points.

Theorem 3.8. Assume that there exists $m_{1}, m_{2} \in \mathbb{L}_{++}^{1}, q_{1}, q_{2} \in \mathbb{L}_{+}^{1}$ and two functions $\varphi_{1}, \varphi_{2}:[0,+\infty) \rightarrow$ $[0,+\infty)$ such that $\mu_{\alpha}\left(m_{1}\right)<1<\mu_{\alpha}\left(m_{2}\right)$ and, for all $u \geq 0$ and a.e. $t \in(0,1)$,

$$
m_{1}(t) u-q_{1}(t) \varphi_{1}(u) \leq f(t, u) \leq m_{2}(t) u+q_{2}(t) \varphi_{2}(u) .
$$

If either

$$
\begin{aligned}
& \varphi_{1}(u)=\circ(\|u\|) \text { as } u \rightarrow \infty, \varphi_{2}(u)=\circ(\|u\|) \text { as } u \rightarrow 0 \\
& \varphi_{1} \text { is nondecreasing and } \varphi_{2} \text { is nondecreasing near } 0,
\end{aligned}
$$

or

$$
\varphi_{1}(u)=\circ(\|u\|) \text { as } u \rightarrow 0 \text { and } \varphi_{2}(u)=\circ(\|u\|) \text { as } u \rightarrow \infty \text {, }
$$

$\varphi_{1}$ is nondecreasing near 0 and $\varphi_{2}$ is nondecreasing,

then FBVP (1.1) admits a positive solution.

Proof. Let, for $i=1,2, F_{i}: P \rightarrow P$ be defined by

$$
F_{i} u(t)=\int_{0}^{1} G(t, s) \varphi_{i}(u(s)) d s .
$$

We have then from Hypothesis (3.12) that, for all $u \in P$

$$
L_{m_{1}} u-F_{1} u \preceq T u \preceq L_{m_{2}} u+F_{2} u,
$$


with

$$
r\left(L_{m_{2}}\right)=1 / \mu_{\alpha}\left(m_{2}\right)<1<r\left(L_{m_{1}}\right)=1 / \mu_{\alpha}\left(m_{1}\right) .
$$

Suppose that (3.13) holds (the case where (3.14) holds can be checked similarly). We have

$$
\frac{\left\|F_{i} u\right\|_{\infty}}{\|u\|_{\infty}}=\sup _{t \in[0,1]} \frac{F_{i} u(t)}{\|u\|_{\infty}} \leq \int_{0}^{1} G(1, s) q_{i}(s) \frac{\varphi_{i}(u(s))}{\|u\|_{\infty}} d s \leq \frac{\varphi_{i}\left(\|u\|_{\infty}\right)}{\|u\|_{\infty}} \int_{0}^{1} G(1, s) q_{i}(s) d s,
$$

which leads to

$$
F_{1} u=\circ(\|u\|) \text { as } u \rightarrow \infty \text { and } F_{2} u=\circ(\|u\|) \text { as } u \rightarrow 0 .
$$

We conclude from Theorem 2.14 that operator $T$ admits a fixed point, which is a positive solution to FBVP (1.1) from iii) of Lemma 3.5.

Example 3.9. Let $a, b \in \mathbb{L}_{++}^{1}$. The function $f(t, u)=a(t) \frac{u(1+|\sin u|)}{1+u}+b(t) \frac{u^{3}(1+|\cos u|)}{2\left(1+u^{2}\right)}$ satisfies (3.12)-(3.14) with

$$
\begin{aligned}
m_{1}(t) & =a(t), m_{2}(t)=b(t), q_{1}(t)=\max (a(t), b(t)), q_{2}(t)=a(t), \\
\varphi_{1}(u) & =2 \max \left(u^{2}, u^{3}\right), \varphi_{2}(u)=\frac{2 u}{1+u} .
\end{aligned}
$$

The Separable Case. We consider now the FBVP

$$
\left\{\begin{array}{l}
D^{\alpha} u(t)+q(t) g(u(t))=0,0<t<1 \\
u(0)=u^{\prime}(0)=u^{\prime}(1)=0
\end{array}\right.
$$

where $q \in \mathbb{L}_{++}^{1}$ and $g:[0,+\infty) \rightarrow[0,+\infty)$ is continuous. Set for $v=0$ or $+\infty$

$$
g_{v}=\lim _{u \rightarrow v} \inf \frac{g(u)}{u}, \quad g^{v}=\lim _{u \rightarrow v} \sup \left(\frac{g(u)}{u}\right) .
$$

Corollary 3.10. If either

or

$$
\inf \left\{\frac{g(u)}{u}: u>0\right\}>\mu_{\alpha}(q)
$$

$$
\sup \left\{\frac{g(u)}{u}: u>0\right\}<\mu_{\alpha}(q)
$$

then FBVP (3.15) admits no positive solution.

Proof. Suppose that Hypothesis (3.16) is satisfied (the other case can be checked similarly) and let $\varepsilon_{0}>0$ be such that $g(u)>\left(\mu_{\alpha}(q)+\varepsilon_{0}\right) u$. We then have $q(t) g(u)>m(t) u$ for all $u>0$ and a.e. $t \in(0,1)$, where $m(t)=\left(\mu_{\alpha}(q)+\varepsilon_{0}\right) q(t)$. Moreover,

$$
\mu_{\alpha}(m)=\mu_{\alpha}\left(\left(\mu_{\alpha}(q)+\varepsilon_{0}\right) q\right)=\frac{\mu_{\alpha}(q)}{\left(\mu_{\alpha}(q)+\varepsilon_{0}\right)}<1 .
$$

Thus, Hypothesis (3.10) of Proposition 3.7 is satisfied and FBVP (3.15) admits no positive solution.

Corollary 3.11. If either

$$
g^{0}<\mu_{\alpha}(q)<g_{+\infty}
$$

or

$$
g^{+\infty}<\mu_{\alpha}(q)<g_{0}
$$

then FBVP (3.15) admits a positive solution. 
Proof. We present the proof in the case where (3.18) holds, the other case is checked similarly. Let $\varepsilon>0$ be such that $\left(g^{0}+\varepsilon\right)<\mu_{\alpha}(q)<\left(g_{+\infty}-\varepsilon\right)$. Then, there exists a positive constant $C_{\infty}$ such that, for all $u \geq 0$,

$$
\left(g_{+\infty}-\varepsilon\right) u-C_{\infty} \leq g(u) \leq\left(g^{0}+\varepsilon\right) u+h(u)
$$

where

$$
h(u)=\max \left(0, g(u)-\left(g^{0}+\varepsilon\right) u\right)
$$

Set

$$
m_{1}=\left(g_{+\infty}-\varepsilon\right) q, m_{2}=\left(g^{0}+\varepsilon\right) q, \varphi_{1}(u)=C_{\infty}, \varphi_{2}(u)=h(u) .
$$

So,

$$
\mu_{\alpha}\left(m_{1}\right)=\mu_{\alpha}\left(\left(g_{+\infty}-\varepsilon\right) q\right)=\frac{\mu_{\alpha}(q)}{\left(g_{+\infty}-\varepsilon\right)}<1<\mu_{\alpha}\left(m_{2}\right)=\mu_{\alpha}\left(\left(g^{0}+\varepsilon\right) q\right)=\frac{\mu_{\alpha}(q)}{\left(g^{0}+\varepsilon\right)}
$$

and

$$
\varphi_{1}(u)=\circ(\|u\|) \text { as } u \rightarrow \infty \text { and } \varphi_{2}(u)=\circ(\|u\|) \text { as } u \rightarrow 0 .
$$

Thus, Hypothesis (3.13) is satisfied and the existence of a positive solution to FBVP (3.15) can be obtained from Theorem 3.8.

\section{Acknowledgements}

The authors thank the anonymous referee for all his valuable comments and suggestions which improved this paper.

\section{REFERENCES}

[1] A. Cabada, G. Wang, Positive solutions of nonlinear fractional differential equations with integral boundary value conditions, J. Math. Anal. Appl. 389 (2012), 403-411.

[2] J. Caballero, I. Cabrera, K. Sadarangani, Positive solutions of nonlinear fractional differential equations with integral boundary value conditions, Abstr. Appl. Anal. 2012 (2012), Article ID 303545.

[3] M. Feng, X. Zhang, W. Ge, New existence results for Higher-order nonlinear fractional differential equations with integral boundary conditions, Bound. Value Probl. 2011 (2011), Article ID 720702.

[4] D. Fu, W. Ding, Existence of positive solutions of third-order boundary value problems with integral boundary conditions in Banach spaces, Adv. Difference Equ. 2013 (2013), Article ID 65.

[5] M. Jia, X. Liu; Three nonnegative solutions for fractional differential equations with integral boundary conditions, Comput. Math. Appl. 62 (2011), 1405-1412.

[6] J. Jin, X. Liu, M. Jia, Existence of positive solutions for singular fractional differential equations with integral boundary conditions, Electron. J. Differential Equations 2012 (2012), Article ID 63.

[7] R. A. Khan, M. Ur Rehman, J. Henderson, Existence and uniqueness of solutions for nonlinear fractional differential equations with integral boundary conditions, Fract. Differ. Calc. 1 (2011), 29-43.

[8] A. Benmezaï, Positive solutions for a second order two point boundary value problem, Commun. Appl. Anal. 14 (2010), 177-190.

[9] A. Benmezaï, W. Esserhane and J. Henderson, Existence of positive solutions for singular second order boundary value problems under eigenvalue criteria, Dyn. Contin. Discrete Impuls. Syst. Ser. A Math. Anal. 20 (2013), 709-725.

[10] A. Benmezaï, J. R. Graef, L. Kong, Positive solutions to a two point singular boundary value problem, Differ. Equ. Appl. 3 (2011), no. 3, 347-373.

[11] A. Benmezaï, John R. Graef, L. Kong, Positive solutions for the abstract Hammerstein equations and applications, Commun. Math. Anal. 16 (2014), 47-65.

[12] D. Guo, V. Lakshmikantaham, Nonlinear Problems in Abstract Cones, Academic Press, San Diego, 1988. 
[13] E. Zeidler, Nonlinear Functional Analysis and its Applications, Vol. I, Fixed Point Theorems, Springer-Verlag, New York, 1986.

[14] J.R.L. Webb, A class of positive linear operators and applications to nonlinear boundary value problems, Topol. Methods Nonlinear Anal. 39 (2012), 221-242.

[15] A. A. Kilbas, H. M. Srivastava, J. J. Trujillo, Theory and Applications of Fractional Differential Equations, North-Holland Mathematics Studies, 204, Elsevier Science B.V., Amsterdam, 2006.

[16] I. Podlubny, Fractional Differential Equations, Academic Press, San Diego, 1999. 